\title{
Final analysis of a phase II study of modified FOLFIRINOX in locally advanced and metastatic pancreatic cancer
}

Stacey M Stein ${ }^{1,7}$, Edward S James ${ }^{1,7}$, Yanhong Deng ${ }^{2}$, Xiangyu Cong ${ }^{2}$, Jeremy S Kortmansky ${ }^{1}$, Jia Li $^{1,3}$, Carol Staugaard ${ }^{1}$, Doddamane Indukala ${ }^{4}$, Ann Marie Boustani ${ }^{4}$, Vatsal Patel ${ }^{4}$, Charles $\mathrm{H} \mathrm{Cha}^{5}$, Ronald R Salem ${ }^{5}$, Bryan Chang ${ }^{6}$, Howard S Hochster ${ }^{1}$ and Jill Lacy*,1

${ }^{1}$ Department of Medicine, Section of Medical Oncology, Yale School of Medicine, 333 Cedar Street, New Haven, CT 06510, USA; ${ }^{2}$ Yale School of Public Health, 300 George Street, New Haven, CT 06510, USA; ${ }^{3}$ VA Connecticut Healthcare System, 950 Campbell Avenue, West Haven, CT 06516, USA; ${ }^{4}$ Department of Diagnostic Radiology, Yale School of Medicine, 333 Cedar Street, New Haven, CT 06510, USA; ${ }^{5}$ Department of Surgery, Yale School of Medicine, 333 Cedar Street, New Haven, CT 06510, USA and ${ }^{6}$ Department of Therapeutic Radiology, Yale School of Medicine, 333 Cedar Street, New Haven, CT 06510, USA

Background: Modifications of FOLFIRINOX are widely used despite the absence of prospective data validating efficacy in metastatic disease (metastatic pancreatic cancer (MPC)) or locally advanced pancreatic cancer (LAPC). We conducted a multicentre phase II study of modified FOLFIRINOX in advanced pancreatic cancer to assess the impact of dose attenuation in MPC and efficacy in LAPC.

Methods: Patients with untreated MPC or LAPC received modified FOLFIRINOX (irinotecan and bolus 5-fluorouracil reduced by 25\%). Adverse events (AEs) were compared with full-dose FOLFIRINOX. Response rate (RR), median progression-free survival (PFS) and median overall survival (OS) were determined.

Results: In total, 31 and 44 patients with LAPC and MPC were enrolled, respectively. In MPC, efficacy of modified FOLFIRINOX was comparable with FOLFIRINOX with RR 35.1\%, OS 10.2 months (95\% Cl 7.65-14.32) and PFS 6.1 months (95\% Cl 5.19-8.31). In LAPC, efficacy was notable with RR 17.2\%, resection rate 41.9\%, PFS 17.8 months (95\% Cl 11.0-23.9) and OS 26.6 months (95\% Cl 16.7, NA). Neutropenia $(P<0.0001)$, vomiting $(P<0.001)$ and fatigue $(P=0.01)$ were significantly decreased. $\left[{ }^{18} \mathrm{~F}\right]-\mathrm{Fluorodeox}-$ yglucose positron emission tomography imaging response did not correlate with PFS or OS.

Conclusions: In this first prospective study of modified FOLFIRINOX in MPC and LAPC, we observed decreased AEs compared with historical control patients. In MPC, the efficacy appears comparable with FOLFIRINOX. In LAPC, PFS and OS were prolonged and support the continued use of FOLFIRINOX in this setting.

Pancreatic ductal carcinoma is a highly lethal malignancy with a five-year survival rate of just $6 \%$ in the United States (Rahib et al, 2014). The incidence of pancreatic cancer is increasing, and in the absence of meaningful advances in prevention or treatment, it is projected that pancreatic cancer will emerge as the second leading cause of cancer-related deaths in the United States by 2030 (Rahib et al, 2014). While surgical resection may be curative, the cure rate is only $5 \%$. Furthermore, up to $80 \%$ of patients have unresectable disease at the time of diagnosis due to distant metastases or vascular involvement (Malik et al, 2012). Between 1997 and 2010,

\footnotetext{
*Correspondence: Professor J Lacy; E-mail: jill.lacy@yale.edu

${ }^{7}$ These two authors contributed equally to this work.
} 
the median survival of patients with metastatic and locally advanced pancreatic cancer (LAPC) enrolled into multiple prospective clinical trials was consistently less than 7 and 11 months, respectively. During this period, gemcitabine remained the standard of care for advanced pancreatic cancer, as multiple randomised phase III trials were unable to demonstrate improved survival with gemcitabine-based combinations compared with gemcitabine monotherapy. In LAPC, the addition of radiotherapy to gemcitabine-based therapy did not convincingly improve outcomes (Hong, 2012).

In 2010, FOLFIRINOX was introduced into clinical practice and heralded as a significant advance in the treatment of metastatic pancreatic cancer (MPC) based on the results of the PRODIGE 4/ACCORD 11 trial. In this randomised prospective phase III study comparing FOLFIRINOX with gemcitabine in patients with MPC with a good performance status, FOLFIRINOX was associated with an unprecedented overall survival (OS) of 11.1 months compared with 6.8 months with gemcitabine, as well as significant improvements in progression-free survival (PFS) and response rate (RR) (Conroy et al, 2011; Gourgou-Bourgade et al, 2013). Moreover, the FOLFIRINOX regimen was associated with an improvement in quality-of-life measures compared with singleagent gemcitabine (Gourgou-Bourgade et al, 2013). However, the significant toxicities of FOLFIRINOX reported in the PRODIGE 4/ ACCORD 11 trial, including neutropenia, fatigue, diarrhoea and vomiting, have tempered enthusiasm for the widespread use of FOLFIRINOX in full doses in community and academic practices. Despite the absence of prospective data regarding efficacy and tolerability, use of ad hoc modifications of FOLFIRINOX has been reported retrospectively by several institutions (Marsh et al, 2015), and ongoing trials with FOLFIRINOX in combination with novel agents often incorporate modifications that have not been piloted or studied.

Given its efficacy in MPC, FOLFIRINOX is now widely used for LAPC. Published experience with this regimen in the locally advanced setting, however, has been confined to retrospective reviews (Hosein et al, 2012; Faris et al, 2013; Khushman et al, 2015). Although the retrospective data are promising, prospective studies with FOLFIRINOX in LAPC have not yet been reported.

To assess the tolerability of FOLFIRINOX in our practice at Smilow Cancer Hospital, we retrospectively analysed all patients with advanced pancreatic cancer treated with FOLFIRINOX at our institution between June 2010 and July 2011(Gunturu et al, 2013). Our analysis suggested that modest initial dose attenuations of bolus 5 -fluorouracil (5FU) and/or irinotecan were associated with excellent tolerability, while efficacy appeared to be comparable with previously reported data (Conroy et al, 2011). Based on our institution's experience, we conducted a prospective phase II open label study to evaluate the efficacy and tolerability of FOLFIRINOX using upfront dose reductions of the $5 \mathrm{FU}$ bolus (25\%) and irinotecan $(25 \%)$ in patients with advanced pancreatic cancer. Herein, we report the final analysis of the efficacy and toxicity of modified FOLFIRINOX in both LAPC and MPC.

\section{MATERIALS AND METHODS}

Design. We conducted a phase II open label single arm multiinstitutional study at Yale's Smilow Cancer Hospital (New Haven, CT, USA), the Smilow Cancer Hospital Care Centers (our regional community-based clinics), the VA Connecticut Healthcare System West Haven Campus (West Haven, CT, USA) and Bridgeport Hospital (Bridgeport, CT, USA) from November 2011 through January 2014. The primary objective of this study was to determine the PFS in patients with MPC and LAPC treated with a doseattenuated modification of FOLFIRINOX. Secondary objectives were to determine objective response rate according to RECIST, OS, and toxicities and to correlate early changes in glucose metabolism using $\left[{ }^{18} \mathrm{~F}\right]$-fluorodeoxyglucose positron emission tomography (FDG-PET) scanning with PFS and OS. The study is registered on the clinical trial web site of the National Cancer Institute (ClinicalTrials.gov identifier: NCT01523457).

Patient selection. Patients with pathologically confirmed, measurable or non-measurable assessable MPC or LAPC (including unresectable and borderline resectable) were eligible for inclusion in this study. Patients were determined to have locally advanced unresectable or borderline resectable disease by the investigators (RRs, CHC) using the imaging criteria established by the National Comprehensive Cancer Network (NCCN) (Callery et al, 2009; Tempero et al, 2010). No prior therapy of any type for advanced disease was allowed. Prior adjuvant chemotherapy or radiotherapy for resected pancreatic adenocarcinoma was allowed if more than 6 months had elapsed since completion of prior therapy and registration. Additional inclusion criteria were as follows: age 18 or older, ECOG performance status of 0 or 1 , no prior oxaliplatin or irinotecan therapy, and adequate haematological, renal and liver function (absolute neutrophil count $\geqslant 1500 / \mu \mathrm{l}$, platelet count $\geqslant 100000 / \mu$ l, haemoglobin $\geqslant 9 \mathrm{~g} \mathrm{dl}^{-1}$, creatinine $<1.5 \times \mathrm{ULN}$ or estimated GFR $>30 \mathrm{ml} \mathrm{min}^{-1}$, bilirubin $<1.5 \times \mathrm{ULN}$, and AST and ALT $<3 \times$ ULN). Patients were excluded if they had grade $\geqslant 2$ peripheral sensory neuropathy, chronic diarrhoea, significant cardiac disease, any serious coexisting medical conditions, or were pregnant or breastfeeding.

All patients provided informed consent. The protocol was approved by institutional review boards at all participating institutions. The study was conducted in accordance with Good Clinical Practice guidelines and the Declaration of Helsinki.

Treatment. Patients were treated with modified FOLFIRINOX every 2 weeks as follows: oxaliplatin $85 \mathrm{mg} \mathrm{m}^{-2}$ infused over $120 \mathrm{~min}$, immediately followed by folinic acid $400 \mathrm{mg} \mathrm{m}^{-2}$ infused over $120 \mathrm{~min}$ with the addition, after $30 \mathrm{~min}$, of irinotecan $135 \mathrm{mg} \mathrm{m}^{-2}$ infused over $90 \mathrm{~min}$, followed by $5 \mathrm{FU} 300 \mathrm{mg} \mathrm{m}^{-2}$ IV bolus, followed by $2400 \mathrm{mg} \mathrm{m}^{-2}$ continuous infusion for $46 \mathrm{~h}$ ( $25 \%$ reduction in bolus $5 \mathrm{FU}$ and irinotecan doses). All patients received pegylated filgrastim with each cycle on day 3 or 4 in the absence of severe leukocytosis. All patients routinely received palonosetron, aprepitant and dexamethasone for emesis prophylaxis. Protocol-specified treatment modifications or delays for treatment-related adverse events were mandated (Conroy et al, 2011). If treatment was held for any treatment-related toxicities for $\geqslant 3$ weeks, protocol therapy was discontinued.

Assessments. Patients were evaluated for toxicities at the start of each cycle with history, physical examination, performance status, complete blood count and metabolic panel. Toxicities were graded according to the National Cancer Institute Common Toxicity Criteria version 3.0. computed tomograph (CAT) scans were obtained after every four cycles. Tumour response was determined according to RECIST 1.1 by independent radiology review. $\left[{ }^{18} \mathrm{~F}\right]$ Fluorodeoxyglucose positron emission tomography scans were obtained at baseline and after two cycles. Metabolic response was categorised into four groups based on \% change in summed SUVs at maximum five sites (including primary when possible): significant response, $\geqslant 50 \%$ decrease; mild response, $<50 \%$ to $\geqslant 25 \%$ decrease; stable disease $<25 \%$ decrease to $\geqslant 25 \%$ increase; progression of disease, $>25 \%$ increase.

Duration of treatment and follow-up. The duration of study treatment was 12 cycles in the MPC cohort and eight cycles in the LAPC cohort. Patients who completed the study treatment phase without disease progression in both cohorts were allowed to enter a post-study treatment phase and continue treatment per protocol guidelines until progression at the discretion of the treating 
physician. Treatment during the study or post-study phase was discontinued for unacceptable toxicity, progression of disease, treating physician's discretion, patient's unwillingness to continue protocol treatment for personal reasons, or pursuit of alternative therapies in the LAPC cohort (surgical resection or radiotherapy). Oxaliplatin was permanently discontinued for grade 3 sensory neuropathy persisting between treatments, and treatment was continued without oxaliplatin. Surgery was pursued in patients deemed candidates for $\mathrm{R} 0$ resection during or after study treatment as determined by the investigators (RRS, CHC). Patients were followed for PFS and OS.

Statistics. Toxicities in both the LAPC and MPC cohorts were compared with the historical control group of patients with metastatic disease treated with standard FOLFIRINOX as reported by Conroy et al (2011). For comparison of patient characteristics and response rate with the historical control group, we included only those patients with MPC. One sample median test (one sample Wilcoxon signed-rank test) for continuous variables and Fisher's exact test for categorical variables were used to compare patient characteristics. Fisher's exact test was used to compare the proportion of grade 3 and 4 adverse events. Progression-free survival and OS in the MPC and LAPC cohorts were summarised using Kaplan-Meier curves. Associations of metabolic response by FDG-PET imaging to PFS and OS were analysed by log-rank test and Fisher's exact test. $P$-value of $<0.05$ was considered statistically significant. Analyses were conducted using SAS 9.3 (Cary, NC, USA).

\section{RESULTS}

Patient characteristics and drug delivery. Between November 2011 and March 2014, 75 patients, including 44 with MPC and 31 with LAPC, were enrolled. The demographics and disease characteristics of the 37 evaluable patients in the MPC cohort in this study and the historical control group treated with standard FOLFIRINOX are shown in Table 1. There were no significant differences in age, sex, performance status, CA19-9 level, pancreatic tumor location or presence of biliary stent in our MPC cohort compared with the historical control group (Conroy et al, 2011). Significantly more patients in our MPC cohort had peritoneal metastases $(37.8 \%$ vs $19.4 \%, P=0.010)$, and there were fewer patients with liver metastases $(54.1 \%$ vs $87.6 \%, P<0.0001)$. Median number of cycles in the MPC cohort was 9 (range 1-46). Twenty-one patients in the MPC cohort did not complete 12 cycles due to progression of disease $(n=16)$, treatment delay for unresolved grade $>1$ diarrhoea $(n=1)$ or infection $(n=3)$ and patient preference $(n=1)$. The median relative dose intensities of bolus 5FU, infusional 5FU, bolus plus infusional 5FU combined, irinotecan and oxaliplatin were $88.9 \%, 90.9 \%, 89.7 \%, 88.9 \%$ and $88.9 \%$, respectively. The percentage of patients who received full doses of bolus 5FU, infusional 5FU, irinotecan and oxaliplatin for all cycles were $70.3 \%, 75.7 \%, 59.5 \%$ and $59.5 \%$, respectively.

The demographics and disease characteristics for the LAPC cohort are shown in Table 2. Median number of cycles in the LAPC cohort was 8 (range 4-21). In 13 patients who underwent surgical resection, the median number of preoperative FOLFIRINOX cycles was 8 (range 4-12) and the median number of combined pre- and postoperative cycles was 10 (range 6-13). Treatment was discontinued prior to completing eight cycles in 15 patients, including four who went on to surgery, five who had treatment delays for unresolved infection $(n=2)$ or grade $>1$ toxicities $(n=3)$, four with stable disease who opted to receive chemoradiation and two who declined further treatment. No patients in the LAPC cohort discontinued study treatment due to progression of disease. The median relative dose intensities of bolus
Table 1. Patient and disease characteristics of the MPC cohort

\begin{tabular}{|c|c|c|c|}
\hline Characteristics & $\begin{array}{l}\text { MPC cohort } \\
(n=37)\end{array}$ & $\begin{array}{l}\text { Historical } \\
(n=171)\end{array}$ & $P$-value ${ }^{a}$ \\
\hline Age, median (range) & $62(50-77)$ & $61(25-76)$ & $0.75^{b}$ \\
\hline Male, $n(\%)$ & $21(56.8)$ & $106(62)$ & $0.58^{c}$ \\
\hline ECOG PS, n (\%) & & & $0.47^{c}$ \\
\hline $\begin{array}{l}0 \\
1\end{array}$ & $\begin{array}{l}17(46.0) \\
20(54.0)\end{array}$ & $\begin{array}{r}64(37.4) \\
106(62.0)\end{array}$ & \\
\hline \multicolumn{4}{|c|}{ Pancreatic tumour location, n/total no. $(\%)^{d}$} \\
\hline $\begin{array}{l}\text { Head } \\
\text { Body }\end{array}$ & $\begin{array}{l}17 / 31(54.8) \\
14 / 31(45.2)\end{array}$ & $\begin{array}{l}67 / 165(40.6) \\
98 / 165(59.4)\end{array}$ & $0.58^{c}$ \\
\hline \multicolumn{4}{|c|}{ Metastatic sites, n/total no. (\%) } \\
\hline $\begin{array}{l}\text { Liver } \\
\text { Peritoneal } \\
\text { Lung } \\
\text { Lymph node }\end{array}$ & $\begin{array}{l}20 / 37(54.1) \\
14 / 37(37.8) \\
12 / 37(32.4) \\
15 / 37(40.5)\end{array}$ & $\begin{array}{r}149 / 170(87.6) \\
33 / 170(19.4) \\
33 / 170(19.4) \\
49 / 170(28.8)\end{array}$ & $\begin{array}{r}<0.0001^{c} \\
0.010^{c} \\
0.059^{c} \\
0.145^{c}\end{array}$ \\
\hline $\begin{array}{l}\text { Level of CA19.9, n/total } \\
\text { no. (\%) }\end{array}$ & & & $0.85^{\star \star}$ \\
\hline $\begin{array}{l}\text { Normal } \\
\text { Elevated },<59 \times \text { ULN } \\
\text { Elevated, } \geqslant 59 \times \text { ULN }\end{array}$ & $\begin{array}{c}4 / 37(10.8) \\
18 / 37(48.7) \\
15 / 37(40.5)\end{array}$ & $\begin{array}{l}24 / 164(14.6) \\
72 / 164(43.9) \\
68 / 164(41.5)\end{array}$ & \\
\hline $\begin{array}{l}\text { Biliary stent, } n / \text { total no. } \\
\text { (\%) }\end{array}$ & & & $0.23^{c}$ \\
\hline $\begin{array}{l}\text { Yes } \\
\text { No }\end{array}$ & $\begin{array}{r}9 / 37(24.3) \\
28 / 37(75.7) \\
\end{array}$ & $\begin{array}{r}27(15.8) \\
144(84.2) \\
\end{array}$ & \\
\hline \multicolumn{4}{|c|}{ 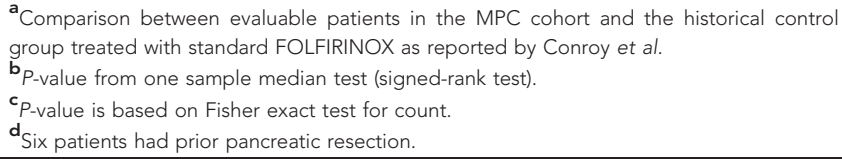 } \\
\hline
\end{tabular}

Table 2. Patient and disease characteristics of the LAPC cohort

\begin{tabular}{|l|c|}
\hline Characteristics & LAPC cohort $(\boldsymbol{n}=\mathbf{3 1})$ \\
\hline Age, median (range) & $63(46-79)$ \\
\hline Male, $n$ (\%) & $20(64.5)$ \\
\hline ECOG PS, $n$ (\%) \\
\hline 0 & $15(48)$ \\
1 & $16(52)$ \\
\hline Pancreatic tumour location, $n$ (\%) \\
\hline Head \\
Body & $27(87)$ \\
\hline Unresectable vs Borderline resectable, $n(\%)$ \\
\hline Unresectable & $4(13)$ \\
\hline Borderline & $20(64.5)$ \\
\hline Level of CA19.9, $n$ (\%) & $11(35.5)$ \\
\hline Normal \\
Elevated, <59 $\times$ ULN & $3(9.6)$ \\
Elevated, $\geqslant 59 \times$ ULN & $4(77.4)$ \\
\hline Biliary stent, $n$ (\%) & $4(12.9)$ \\
\hline Yes & $17(54.8)$ \\
No & $14(45.2)$ \\
\hline
\end{tabular}

$5 \mathrm{FU}$, infusional $5 \mathrm{FU}$, bolus plus infusional $5 \mathrm{FU}$ combined, irinotecan and oxaliplatin were $88.9 \%, 90.4 \%, 88.9 \%, 88.9 \%$ and $88.9 \%$, respectively, in the LAPC cohort. The percentage of patients who received full doses of bolus 5FU, infusional 5FU, irinotecan and oxaliplatin for all cycles were $74.2 \%, 83.9 \%, 67.7 \%$ and $67.7 \%$, respectively.

Efficacy in the MPC cohort. Seven of 44 patients with MPC were excluded from efficacy analysis due to voluntary withdrawal from 
the study for reasons unrelated to disease progression or toxicity prior to completing four cycles to reach the first efficacy assessment (one patient was not treated, and three and four patients received one and two cycles, respectively). At the time of this analysis, 29 patients had expired and eight patients were alive. Three patients remained on treatment. The objective RR by independent radiology review in evaluable patients was $35.1 \%$ and was not significantly different from the response rate in evaluable patients in the FOLFIRINOX-treated historical control group (36.3\%, $P=0.82$ ) (Table 3). Similarly, the RR of $30.2 \%$ for all treated patients (including invaluable patients) did not differ from the $\mathrm{RR}$ in the intent-to-treat FOLFIRINOX-treated historical control group $(30.1 \%, P=0.93)$. The disease control rate was not significantly different from the historical control group $(86.5 \% \mathrm{v}$ $82.2 \%)$.

The PFS rates at 6,12 and 18 months were 54\%, 14\% and 9\%, respectively (Table 4 and Figure 1). The median PFS was 6.1 months (95\% CI 5.19-8.31) (Table 4 and Figure 1). The OS rates at 6,12 and 18 months were $81 \%, 38 \%$ and $22 \%$, respectively, in the MPC cohort (Table 4). The median OS was 10.2 months (95\% CI 7.65-14.32). Three patients (8\%) have survived for more than 3 years (Table 4, Figure 1).

Efficacy in the LAPC cohort. Two of 31 patients with LAPC were excluded from efficacy analysis because they did not complete four cycles to reach the first efficacy assessment (one due to cholecystitis with abscess and one due to cerebrovascular accident). Nineteen patients remained alive and 12 patients had not had disease progression at the time of the analysis. The response rate was $17.2 \%$ with $100 \%$ having disease control (Table 3 ). The PFS rates at 6, 12 and 18 months were $97 \%, 69 \%$ and $62 \%$, respectively (Table 4). The median PFS was 17.8 months (95\% CI 11.0-23.9) (Table 4 and Figure 1). The OS rates at 6,12 and 18 months were $100 \%, 86 \%$ and $62 \%$, respectively (Table 4 ). The median OS was 26.6 months (95\% CI 16.7, NA) (Table 4 and Figure 1).

In patients with stable or responding disease, 11 received chemoradiotherapy, 7 underwent surgery and 6 received chemoradiotherapy followed by surgery. Thirteen patients in the LAPC cohort (41.9\%) had surgical resection; seven patients initially had borderline resectable and six had unresectable disease. All patients who underwent surgery had R0 resections. The post-treatment

Table 3. Response in LAPC and MPC cohorts

\begin{tabular}{|c|c|c|c|c|}
\hline & $\begin{array}{l}\text { LAPC cohort } \\
(n=29)\end{array}$ & $\begin{array}{l}\text { MPC cohort } \\
(n=37)\end{array}$ & $\begin{array}{l}\text { Historical } \\
(n=146)\end{array}$ & $P$-value ${ }^{a}$ \\
\hline \multicolumn{5}{|c|}{ Response, $n(\%)$} \\
\hline CR & $0(0)$ & $0(0)$ & $1(0.7)$ & \\
\hline PR & $5(17.2)$ & $13(35.1)$ & $53(36.3)$ & \\
\hline SD & 24 (82.7) & 19 (51.5) & 66 (45.2) & \\
\hline PD & $0(0)$ & $5(13.5)$ & $26(17.8)$ & \\
\hline$C R+P R$ & $5(17.2)$ & $13(35.1)$ & $54(37.0)$ & $0.87^{b}$ \\
\hline $\mathrm{CR}+\mathrm{PR}+\mathrm{SD}$ & $29(100)$ & 32 (86.5) & $120(82.2)$ & $0.67^{b}$ \\
\hline
\end{tabular}

pathologic stages (and preoperative treatment) were as follows: ypT0N0 stage 0, one patient (chemotherapy); ypT1N0 stage I, two patients (chemotherapy, $n=1$; chemotherapy followed by chemoradiotherapy, $n=1$ ); ypT3N0, six patients (chemotherapy, $n=3$; chemotherapy followed by chemoradiotherapy, $n=3)$; and ypT3N1 stage IIB, four patients (chemotherapy, $n=2$; chemotherapy followed by chemoradiotherapy, $n=2$ ). At the time of the analysis, 10 of 13 patients who underwent surgery were alive, and 6 had no evidence of recurrent disease.

Metabolic response by FDG-PET imaging. We performed an exploratory analysis of the association between early metabolic response by FDG-PET imaging and PFS and OS. In the metastatic cohort, eight patients were excluded from this analysis due to negative baseline FGD-PET scan or failure to obtain the follow-up scan. Among the 29 patients who were evaluated with baseline and follow-up FDG PET scans after two cycles, 24 patients (83\%) had a metabolic response, including 14 patients (48\%) with significant response and 10 patients (34\%) with mild response. Two patients had stable disease and three patients had progression of disease. In the LAPC cohort, six patients were not evaluable. Of the 23 patients who were evaluable, 12 patients (52\%) had a metabolic response, including eight major responses (35\%) and four (17\%) minor responses. Ten patients had stable disease and one patient had progression of disease.

There was no statistical association between metabolic response (combined significant and mild response) and either PFS or OS for either cohort (for metastatic cohort: $P$-values of 0.55 and 0.27 based on Fisher's exact test for count, 0.42 and 0.42 based on logrank test; for locally advanced cohort: $P$-values of 0.37 and 0.68 based on Fisher's exact test for count, 0.44 and 0.43 based on logrank test). Similarly, there was no statistical association between significant metabolic response and PFS or OS for either cohort (for metastatic cohort: $P$-values of 0.60 and 0.65 based on Fisher's exact test for count, 0.88 and 0.69 based on log-rank test; for locally advanced cohort: $P$-values of 0.18 and 0.22 by Fisher's exact test for count, 0.19 and 0.09 based on log-rank test).

Second-line chemotherapy. In the MPC cohort, three patients remained on study treatment, 21 patients received second-line chemotherapy with gemcitabine $(n=5)$ or gemcitabine-based regimen $(n=16)$ and 13 patients did not receive second-line therapy. In the LAPC cohort, 16 of 18 patients who had progression of disease received second-line chemotherapy with gemcitabine-based chemotherapy $(n=11)$ or re-introduction of FOLFOX $(n=1)$, FOLFIRI $(n=1)$ or FOLFIRINOX $(n=1)$; two patients did not receive second-line chemotherapy.

Adverse events. One of 75 patients did not receive treatment and was excluded from toxicity analysis. Treatment-related grade 3 and 4 adverse events observed in our study and in the historical control group treated with standard FOLFIRINOX are summarised in Table 5. As we did not observe differences in the spectrum or incidence of toxicities between the MPC and LAPC patients, we combined these cohorts for a sample size of 74 patients. There were no treatment-related deaths or non-haematologic grade 4 toxicities attributed to modified FOLFIRINOX. Grade 3 and 4 treatmentrelated adverse events were as follows: diarrhoea,16.2\% (grade 3

Table 4. Progression-free and overall survival in MPC and LAPC cohorts

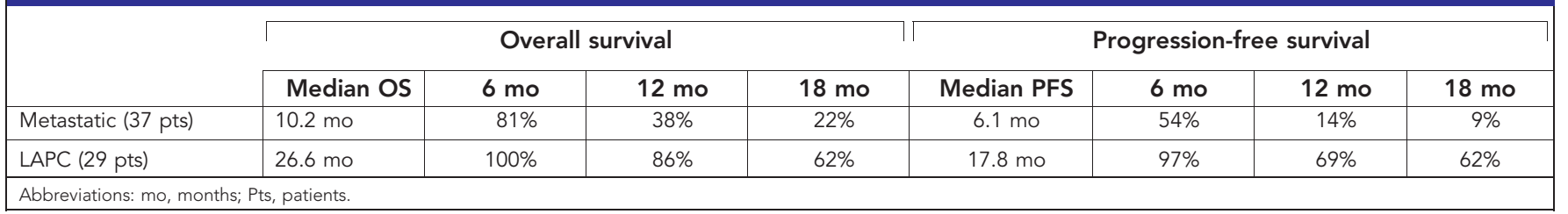



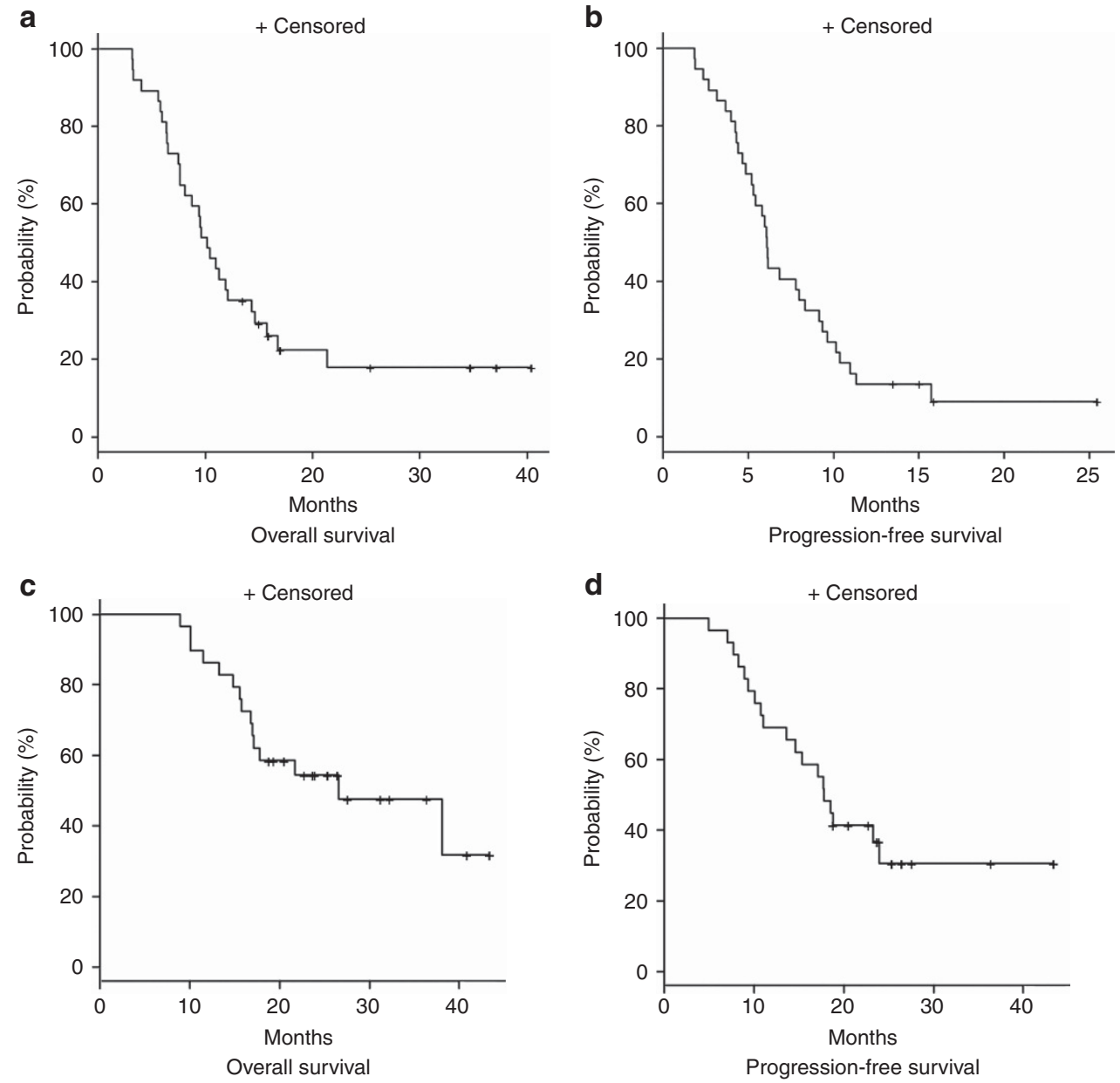

Figure 1. The Kaplan-Meier curve for overall survival and progression-free survival in the metastatic cohort (A, B) and in the locally advanced cohort (C, D).

Table 5. Grade 3/4 adverse events in MPC and LAPC cohorts and historical control

\begin{tabular}{|c|c|c|c|}
\hline Adverse event & $\begin{array}{c}\text { MPC \& LAPC } \\
(N=74) \\
\text { no. of pts/total } \\
\text { no. }(\%)\end{array}$ & $\begin{array}{c}\begin{array}{c}\text { Historical } \\
(N=171)\end{array} \\
\text { no. of pts/total } \\
\text { no. }(\%)\end{array}$ & $P$-value ${ }^{a}$ \\
\hline \multicolumn{4}{|l|}{ Haematologic } \\
\hline Neutropenia & $9 / 74(12.2)$ & $75 / 164(45.7)$ & $<0.0001$ \\
\hline Thrombocytopenia & $7 / 74(9.5)$ & $15 / 165(9.1)$ & 0.83 \\
\hline Anaemia & $4 / 74(5.4)$ & 13/166 (7.8) & 0.66 \\
\hline Febrile neutropenia & $3 / 74(4.1)$ & 9/166 (5.4) & 0.79 \\
\hline \multicolumn{4}{|l|}{ Nonhaematologic } \\
\hline Diarrhoea & $12 / 74(16.2)$ & $21 / 165(12.7)$ & 0.38 \\
\hline Fatigue & $9 / 74(12.2)$ & $39 / 165(23.6)$ & 0.02 \\
\hline $\begin{array}{l}\text { Alanine aminotransferase } \\
\text { (ALT) increased }\end{array}$ & $3 / 74(4.1)$ & $12 / 165(7.3)$ & 0.37 \\
\hline Thromboembolic event & $3 / 74(4.1)$ & $11 / 166(6.6)$ & 0.48 \\
\hline $\begin{array}{l}\text { Peripheral sensory } \\
\text { neuropathy }\end{array}$ & $2 / 74(2.7)$ & 15/166 (9.0) & 0.06 \\
\hline Vomiting & $2 / 74(2.7)$ & $24 / 166(14.5)$ & 0.001 \\
\hline \multicolumn{4}{|c|}{$\begin{array}{l}\text { P-value is based on Fisher's exact test. } \\
\text { aComparison between MPC and LAPC cohorts and the historical control group treated with } \\
\text { standard FOLFIRINOX as reported by Conroy et al. }\end{array}$} \\
\hline
\end{tabular}

only); neutropenia and fatigue, $12.2 \%$; thrombocytopenia, $9.5 \%$; anaemia, 5.4\%; ALT elevated, thromboembolism and febrile neutropenia, 4.1\%; vomiting and peripheral neuropathy, $2.7 \%$.
The incidence of grade 3 or 4 neutropenia (12.2\% vs $45.7 \%$, $P<0.0001)$, vomiting $(2.7 \%$ vs $14.5 \%, P=0.001)$ and fatigue (12.2\% vs $23.6 \%, P=0.02$ ) was significantly decreased in our study compared with the historical control group treated with standard FOLFIRINOX (Conroy et al, 2011). There was a nonsignificant trend towards a lower incidence of peripheral sensory neuropathy (2.7\% vs $9.0 \%, P=0.06)$.

\section{DISCUSSION}

FOLFIRINOX has been heralded as a major advance in the treatment of advanced pancreatic cancer and is now widely used in patients with both metastatic and LAPC. However, the toxicities associated with standard FOLFIRINOX have prompted widespread use of ad hoc modifications of this regimen in academic centres and community practices. Although single institution retrospective experiences suggest that modest dose attenuations improve tolerability while maintaining efficacy, prospective data validating comparable efficacy and diminished toxicity have not previously been reported. Moreover, there are no prospective data regarding the efficacy of FOLFIRINOX in patients with non-metastatic locally advanced disease.

We report the results of our prospective multiinstitutional phase II open label study to evaluate the efficacy and tolerability of modified FOLFIRINOX using upfront dose reductions of the 5FU bolus (25\%) and irinotecan (25\%) in patients with advanced pancreatic cancer. The rationale for these specific dose reductions was based on a retrospective review of all patients treated with 
FOLFIRINOX at our institution between June 2010 and July 2011 (Gunturu et al, 2013). In our retrospective analysis, the majority of patients received attenuated doses of bolus 5FU and irinotecan with the first and subsequent cycles, and the median relative dose intensity of bolus FU and irinotecan was lower than those reported in the FOLFIRINOX-treated group reported by Conroy et al (2011). Our findings suggested that upfront dose attenuations of bolus 5FU and irinotecan improved tolerability without compromising efficacy and provided the rationale for the dose attenuations utilised in this trial (Gunturu et al, 2013).

We have now shown in this prospective study that the efficacy of modified FOLFIRINOX with upfront dose attenuations of bolus $5 \mathrm{FU}$ and irinotecan is comparable with standard FOLFIRINOX in patients with MPC. In addition, we have demonstrated a notable PFS, OS and resection rate in patients with LAPC. Moreover, the patient characteristics of our MPC cohort were similar to the patient characteristics treated with standard FOLFIRINOX. Of note, the incidence of peritoneal carcinomatosis, which confers a particularly poor prognosis in MPC (Takahara et al, 2015), was significantly higher in our MPC patients compared with the historical control group ( $37 \%$ vs $19.4 \%, P=0.010$ ).

Gemcitabine with nab-paclitaxel is emerging as an alternative to FOLFIRINOX in the initial treatment of MPC based on its superior efficacy compared with gemcitabine (Von Hoff et al, 2013; Goldstein et al, 2015). The recent updated analysis of the phase III MPACT trial reported an OS of 8.7 months with $4 \%$ of patients surviving long term (more than 3 years) (Goldstein et al, 2015). Thus, the OS of 10.2 months in our patient population treated with modified FOLFIRINOX, with $8 \%$ of patients surviving more than 3 years, is at least comparable with gemcitabine with nab-paclitaxel.

In this first prospective study of FOLFIRINOX in LAPC, the efficacy of this regimen is noteworthy. Both categories of locally advanced disease, unresectable and borderline, were included in this study, as the approach to their management in recent years has converged with widespread use of upfront chemotherapy. Although FOLFIRINOX is clearly superior to gemcitabine in MPC, there are limited prospective data regarding efficacy and tolerability of FOLFIRINOX in LAPC. In the initial phase II trial of FOLFIRINOX in advanced pancreatic cancer only 11 patients had locally advance disease with RR 7\% and median OS 15.7 months (Conroy et al, 2005). The PFS and OS of 17.8 and 26.6 months, respectively, in our study surpass previously published reports from randomised trials with chemotherapy or chemoradiotherapy. For example, in the GERGOR/GISCAD trial comparing gemcitabine with gemcitabine/ oxaliplatin, OS in the LAPC cohort was just 10.3 months in both arms (Louvet et al, 2005). In the ECOG trial comparing conventional gemcitabine, gemcitabine FDR and gemcitabine/oxaliplatin in advanced pancreatic cancer, the PFS and OS were 5.4 and 9.2 months, respectively, in the LAPC cohort, with no difference between any of the regimens (Poplin et al, 2009). In the LAP-07 study, which examined the role of radiation following induction gemcitabine with or without erlotinib in LAPC, OS was 11.9 and 13.6 months, respectively (Hammel et al, 2013). Remarkably, the PFS and OS in LAPC in our study trial exceeded that reported for patients with resectable pancreatic cancer undergoing adjuvant gemcitabine (OS 22.8 months) (Oettle et al, 2013) or 5FU (23.6 months) (Neoptolemos et al, 2010). Although our study was not designed to compare the outcomes of patients with borderline resectable $v s$ locally advanced unresectable disease, we observed longer survival in the borderline cohort ( $82 \%$ vs $50 \%$ at 18 months).

Thirteen patients with LAPC $(41.9 \%)$ on our study went on to subsequent R0 resection; six of these patients had radiation after modified FOLFIRINOX and before surgery. The outcome of patients who underwent surgery was noteworthy. Median OS has not been reached (median follow-up 24 months). Ten patients were alive at the time of the analysis, and $82 \%$ were alive at 18 months. Given the small number of patients who received FOLFIRINOX only vs
FOLFIRINOX and radiotherapy prior to surgery, we are unable to draw any conclusions about the impact of preoperative radiation after modified FOLFIRINOX. In the LAP-07 study, radiation after induction gemcitabine was not superior to continuing gemcitabine in the patients with LAPC who had not progressed on gemcitabine (OS 15.3 and 16.5 months, respectively) (Hammel et al, 2013). Studies examining the role of radiation in LAPC patients who have received a FOLFIRINOX regimen have not yet been published, and the benefit of radiation in this setting remains unknown.

Modified FOLFIRINOX was well tolerated in both cohorts. We observed no treatment-related deaths or non-haematologic grade 4 toxicities. Compared with standard FOLFIRINOX, we observed a significantly lower incidence of grade 3 and 4 neutropenia, vomiting and fatigue with modified FOLFIRINOX. Although we observed no incidents of grade 4 diarrhoea, we did not observe a lower overall incidence of grade 3 and 4 diarrhoea compared with standard FOLFIRINOX as reported by Conroy et al (2011). The improved tolerability of modified FOLFIRINOX may be related to the dose modifications, routine use of growth factor support and the routine inclusion of aprepitant in the antiemetic regimen.

In this study, we explored the utility of early metabolic response by FGD-PET imaging in predicting outcome as early metabolic response has been shown to correlate with survival in other solid tumours (Ott et al, 2006; Day et al, 2011). The response rate by FDG-PET imaging was high in the MPC cohort (83\%), and there was no correlation between metabolic response (either $\geqslant 50 \%$ or $\geqslant 25 \%$ decrease in SUV) and PFS or OS in this cohort. Similarly, although the response rate by FGD-PET imaging was lower in the LAPC cohort (52\%), we were unable to demonstrate a correlation between metabolic response and either PFS or OS in patients with locally advanced disease. Given the small sample size of our study, further studies of the utility of serial metabolic imaging in pancreatic cancer may be warranted. However, our results suggest that the use of FGD-PET imaging for response assessment in pancreatic cancer is not justified in routine clinical practice.

We acknowledge that observations from single-arm phase II results should be considered with caution, given the limitations of sample size, possible enrollment bias and patient selection. In addition, the composition of the LAPC cohort was heterogeneous with inclusion of patients with borderline resectable disease. Also, some patients subsequently received radiation, and this study was not designed to assess the impact of radiation in this patient population. Our findings are reassuring, albeit not definitive, that the modifications of FOLFIRINOX used in this study do not appear to impact efficacy in MPC and are associated with a favourable outcome in LAPC.

\section{CONCLUSIONS}

In this first prospective study to evaluate the tolerability and efficacy of a dose-attenuated FOLFIRINOX in MPC and LAPC, the $\mathrm{RR}$ in MPC is not significantly different from the RR reported by Conroy et al using standard FOLFIRINOX. Progression-free survival and OS were also comparable. When given with pegylated filgrastim and aprepitant, modified FOLFIRINOX is associated with significantly less neutropenia, vomiting and fatigue compared with standard FOLFIRINOX. The results of our study suggest that the modifications of FOLFIRINOX used in this prospective trial do not negatively impact efficacy in patients with MPC while improving tolerability. Thus, incorporation of these modifications, with routine use of pegylated filgrastim, can be considered an option for patients similar to those treated at our institution, when balancing toxicity and efficacy.

In this first prospective trial of a FOLFIRINOX regimen in LAPC, the efficacy of this regimen is noteworthy and supports the continued use of this regimen in locally advanced disease. 


\section{ACKNOWLEDGEMENTS}

We greatly appreciate the contributions of Neal Fischbach, Thomas Fynan, Victor Chang, Kevin Jain and Kristin Kaley in the recruitment and care of patients participating in this study. We are grateful for the generous support of the Koenigsberg family in conducting this research. This work was supported by the Yale Cancer Center and US National Cancer Institute (P30CA016359).

\section{CONFLICT OF INTEREST}

The authors declare no conflict of interest.

\section{REFERENCES}

Callery M, Chang K, Fishman E, Talamonti MS, William Traverso L, Linehan DC (2009) Pretreatment assessment of resectable and borderline resectable pancreatic cancer: Expert Consensus Statement. Ann Surg Oncol 16(7): $1727-1733$

Conroy T, Desseigne F, Ychou M, Bouché O, Guimbaud R, Bécouarn Y, Adenis A, Raoul JL, Gourgou-Bourgade S, de la Fouchardière C, Bennouna J, Bachet JB, Khemissa-Akouz F, Péré-Vergé D, Delbaldo C, Assenat E, Chauffert B, Michel P, Montoto-Grillot C, Ducreux M. Groupe Tumeurs Digestives of Unicancer; PRODIGE Intergroup (2011) FOLFIRINOX versus gemcitabine for metastatic pancreatic cancer. N Engl J Med 364(19): 1817-1825.

Conroy T, Paillot B, Francois E, Bugat R, Jacob JH, Stein U, Nasca S, Metges JP, Rixe O, Michel P, Magherini E, Hua A, Deplanque G (2005) Irinotecan plus oxaliplatin and leucovorin-modulated fluorouracil in advanced pancreatic cancer - a Groupe Tumeurs Digestives of the Federation Nationale des Centres de Lutte Contre le Cancer study. J Clin Oncol 23(6): $1228-1236$.

Day F, Link E, Ngan S, Leong T, Moodie K, Lynch C, Michael M, Winton Ed, Hogg A, Hicks RJ, Heriot A (2011) FDG-PET metabolic response predicts outcomes in anal cancer managed with chemoradiotherapy. Br J Cancer 105(4): 498-504.

Faris J, Blaszkowsky L, McDermott S, Guimaraes AR, Szymonifka J, Huynh MA, Ferrone CR, Wargo JA, Allen JN, Dias LE, Kwak EL, Lillemoe KD, Thayer SP, Murphy JE, Zhu AX, Sahani DV, Wo JY, Clark JW, Fernandezdel Castillo C, Ryan DP, Hong TS (2013) FOLFIRINOX in locally advanced pancreatic cancer: the Massachusetts General Hospital Cancer Center experience. Oncologist 18(5): 543-548.

Goldstein D, El-Maraghi R, Hammel P, Heinemann V, Kunzmann V, Sastre J, Scheithauer W, Siena S, Tabernero J, Teixeira L, Tortora G, Van Laethem JL, Young R, Penenberg DN, Lu B, Romano A, Von Hoff DD (2015) nabPaclitaxel plus gemcitabine for metastatic pancreatic cancer: long-term survival from a phase III trial. J Natl Cancer Inst 107(2): pii: dju413.

Gourgou-Bourgade S, Bascoul-Mollevi C, Desseigne F, Ychou M, Bouché O, Guimbaud R, Bécouarn Y, Adenis A, Raoul JL, Boige V, Bérille J, Conroy $\mathrm{T}$ (2013) Impact of FOLFIRINOX compared with gemcitabine on quality of life in patients with metastatic pancreatic cancer: results from the PRODIGE 4/ACCORD 11 randomized trial. J Clin Oncol 31(1): 23-29.

Gunturu K, Yao X, Cong X, Thumar JR, Hochster HS, Stein SM, Lacy J (2013) FOLFIRNOX for locally advanced and metastatic pancreatic cancer: single institution retrospective review of efficacy and toxicity. Med Oncol 30(1): 361-367.

Hammel P, Huguet F, Van Laethem J, Goldstein D, Glimelius B, Artru P, Borbath I, Bouche O, Shannon J, Andre T, Mineur L, Chibaudel B, Bonnetain F, Louvet C (2013) Comparison of chemoradiotherapy and chemotherapy in patients with a locally advanced pancreatic cancer controlled after 4 months of gemcitabine with or without erlotinib: final results of the international phase III LAP 07 study. J Clin Oncol 31(suppl): abstr LBA4003.

Hong T (2012) A matter of timing: is there a role for radiation in locally advanced pancreatic cancer, and if so, when? Education Book for ASCO 2012 Annual Meeting, ASCO 2012 Meeting: Chicago, IL, USA, 2012.

Hosein P, Macintrye J, Kawamura C, Maldonado JC, Ernani V, Loaiza-Bonilla A, Narayanan G, Ribeiro A, Portelance L, Merchan JR, Levi JU, RochaLima CM (2012) A retrospective study of neoadjuvant FOLFIRINOX in unresectable or borderline-resectable locally advanced pancreatic adenocarcinoma. BMC Cancer 12: 199-205.

Khushman M, Dempsey N, Cudris Maldonado J, Loaiza-Bonilla A, Velez M, Carcas L, Dammrich D, Hurtado-Cordovi J, Parajuli R, Pollack T, Harwood AP, Macintyre J, Tzeng CW, Merchan JR, Restrepo MH, Akunyili II, Ribeiro A, Narayanan G, Portelance L, Sleeman D, Levi JU, Rocha Lima CM, Hosein PJ (2015) Full dose neoadjuvant FOLFORINOX is associated with prolonged survival in patients with locally advanced pancreatic adenocarcinoma. Pancreatology 15: S1424-S3903.

Louvet C, Labiance R, Hammel P, Lledo G, Zampino MG, André T, Zaniboni A, Ducreux M, Aitini E, Taïeb J, Faroux R, Lepere C, de Gramont A. GERCOR; GISCAD (2005) Gemcitabine in combination with oxaliplatin compared with gemcitabine alone in locally advanced or metastatic pancreatic cancer: results of a GERCOR and GISCAD phase III trial. J Clin Oncol 23(15): 3509-3516.

Malik N, Salerno May K, Chandrasekhar R, Wee W, Flaherty L, Iyer R, Gibbs J, Kuvshinoff B, Wilding G, Warren G, Yang GY (2012) Treatment of locally advanced unresectable pancreatic cancer: a 10-year experience. J Gastrointest Oncol 3(4): 326-334.

Marsh R, Talamonti M, Katz M, Herman JM (2015) Pancreatic cancer and FOLFIRINOX: a new era and new questions. Cancer Med 4(6): 853-863.

Neoptolemos J, Stocken D, Bassi C, Ghaneh P, Cunningham D, Goldstein D, Padbury R, Moore MJ, Gallinger S, Mariette C, Wente MN, Izbicki JR, Friess H, Lerch MM, Dervenis C, Oláh A, Butturini G, Doi R, Lind PA, Smith D, Valle JW, Palmer DH, Buckels JA, Thompson J, McKay CJ, Rawcliffe CL, Büchler MW. European Study Group for Pancreatic Cancer (2010) Adjuvant chemotherapy with fluorouracil plus folinic acid vs gemcitabine following pancreatic cancer resection: a randomized controlled trial. JAMA 304(10): 1073-1081.

Oettle H, Neuhaus P, Hochhaus A, Hartmann JT, Gellert K, Ridwelski K, Niedergethmann M, Zülke C, Fahlke J, Arning MB, Sinn M, Hinke A, Riess H (2013) Adjuvant chemotherapy with gemcitabine and long-term outcomes among patients with resected pancreatic cancer: the CONKO001 randomized trial. JAMA 310(14): 1473-1481.

Ott K, Weber W, Lordick F, Becker K, Busch R, Herrmann K, Wieder H, Fink U, Schwaiger M, Siewert JR (2006) Metabolic imaging predicts response, survival, and recurrence in adenocarcinomas of the esophagogastric junction. J Clin Oncol 24(29): 4692-4698.

Poplin E, Feng Y, Berlin J, Rothenberg ML, Hochster H, Mitchell E, Alberts S, O’Dwyer P, Haller D, Catalano P, Cella D, Benson 3rd AB (2009) Phase III randomized study of gemcitabine and oxaliplatin versus gemcitabine (fixed-dose rate infusion) compared with gemcitabine (30-minute infusion) in patients with pancreatic carcinoma E6201: a trial of the Eastern Cooperative Oncology Group. J Clin Oncol 27(23): 3778-3785.

Rahib L, Smith B, Alzenberg R, Rosenzweig AB, Fleshman JM, Matrisian LM (2014) Projecting cancer incidence and deaths to 2030: the unexpected burden of thyroid, liver, and pancreas cancers in the United States. Cancer Res 74(11): 2913-2921.

Takahara N, Isayama H, Nakai Y, Sasaki T, Saito K, Hamada T, Mizuno S, Miyabayashi K, Mohri D, Kogure H, Matsubara S, Yamamoto N, Hirano K, ijichi H, Tateishi K, Tada M, Koike K (2015) Pancreatic cancer with malignant ascites: clinical features and outcomes. Pancreas 44(3): 380-385.

Tempero M, Arnoletti J, Behrman S, Ben-Josef E, Benson 3rd AB, Berlin JD, Cameron JL, Casper ES, Cohen SJ, Duff M, Ellenhorn JD, Hawkins WG, Hoffman JP, Kuvshinoff 2nd BW, Malafa MP, Muscarella 2nd P, Nakakura EK, Sasson AR, Thayer SP, Tyler DS, Warren RS, Whiting S, Willett C, Wolff RA. NCCN Pancreatic Adenocarcinoma (2010) Pancreatic adenocarcinoma clinical practice guidelines in oncology. J Natl Compr Canc Netw 8(9): 972-1017.

Von Hoff DD, Ervin T, Arena FP, Chiorean EG, Infante J, Moore M, Seay T, Tjulandin SA, Ma WW, Saleh MN, Harris M, Reni M, Dowden S, Laheru D, Bahary N, Ramanathan RK, Tabernero J, Hidalgo M, Goldstein D, Van Cutsem E, Wei X, Iglesias J, Renschler MF (2013) Increased survival in pancreatic cancer with nab-paclitaxel plus gemcitabine. N Engl J Med 369(18): 1691-1703.

This work is published under the standard license to publish agreement. After 12 months the work will become freely available and the license terms will switch to a Creative Commons AttributionNonCommercial-Share Alike 4.0 Unported License. 